\title{
Estimation of Turbine Blade Natural Frequencies from Casing Pressure and Vibration Measurements
}

\author{
*Gareth L. Forbes ${ }^{(1)}$ Robert B. Randall ${ }^{(2)}$ \\ ${ }^{(1)}$ Department of Mechanical Engineering, Curtin University. Perth, WA Australia \\ ${ }^{(2)}$ School of Mechanical and Manufacturing Engineering, University of New South Wales. \\ Sydney, NSW Australia.
}

\begin{abstract}
:
Non-contact measurement of gas turbine rotor blade vibration is a non-trivial task, with no method available which achieves this aim without some significant draw-backs. This paper presents a truly non-contact method to estimate rotor blade natural frequencies from casing vibration measurements at a single engine operating speed. An analytical model is derived to simulate the internal casing pressure in a turbine engine including the effects of blade vibration on this pressure signal. It is shown that the internal pressure inside a turbine contains measureable information about the rotor blade natural frequencies and in-turn the casing vibration response also contains this information. The results presented herein show the residual, pressure and casing vibration, spectrum can be used to determine the rotor blade natural frequencies with validation provided for the analytical model by experimental measurements on a simplified test rig. A simulated blade fault in one of the rotor blades is introduced with successful estimation of the simulated faulty blade natural frequency.
\end{abstract}

\section{Keywords:}

Blade vibration; gas turbine; casing vibration; blade natural frequency; condition monitoring

\section{Introduction:}

It has been reported that blade faults in gas turbines cause up to $42 \%$ of engine failures [1], the highest of all failure modes. Due to the rotor blades passing through unsteady pressure distributions inside a gas turbine engine, blade vibration is unavoidable and inherent in turbine operation. This inherent blade vibration provides an opportunity to use the blade vibration response to measure any degradation of the rotor blades and thus predict the onset of blade faults and mitigate the likelihood of unexpected failures. Blade faults often manifest themselves as changes in the structural response of the blade, allowing for condition 
monitoring of the blades from measuring changes in the natural frequency. Measurement of turbine blade vibration during engine operation is however not a trivial task.

The difficulty in measuring blade vibration within a gas turbine originates primarily due to the rotating domain of the turbine, and the harsh conditions experienced by any sensors which are placed in the gas path. Direct measurement of rotor blade vibration during engine design verification is almost exclusively undertaken with the use of strain gauges mounted on individual blades. This however is not practical to implement outside of the design verification stage, and is not applied in the operational use of gas turbine engines, thus noncontact vibration measurement techniques have been sought. Non-contact measurement of blade vibration has only been implemented successfully, in an operational environment, with the use of blade tip timing (BTT) techniques. BTT vibration measurement has been used for either direct or indirect measurement of blade vibration. Direct methods seek to measure the actual blade response at a given running speed using multiple probes located around a single blade stage periphery, such as the method outlined in Ref. [2]. Indirect BTT seek to measure the rotor blade natural frequencies during operation. This method was first introduced in the 1970's [3], with improvements to the original method being made more recently [4]. These indirect BTT techniques however require the engine to be swept over a range of speeds in order to measure the rotor blade natural frequencies.

Recently, work undertaken by the authors of this paper has suggested that internal pressure and casing vibration measurements contain information about the vibration of the rotor blades, and indeed measureable information about the rotor blade natural frequencies, [5-7]. Another recent study [8], found that the casing pressure spectrum contained sidebands around some multiples of shaft rotational speed at frequencies at which rotor blades on a simplified test rig were excited. It is the measurement of the sidebands around the shaft rotational speed in the pressure and vibration spectrum which will be exploited in this paper to form an estimate of the rotor blade natural frequencies.

The use of casing vibration measurements would be particularly powerful in the determination of rotor blade vibration information as it is a true non-contact method without any need for the sensors to alter the engine or lie within the gas path. In addition to the work by the authors in Refs. [5-7], only one other research work is known to have previously attempted to find a correlation between casing vibration and blade condition [9]. In that work Mathioudakis et al [9] used an inverse filtering technique to reconstruct the internal pressure signal within a gas turbine from the measured casing vibrations by constructing transfer functions between the two signals. That work attempted to demonstrate that the internal pressure signal and casing vibrations are indeed correlated to one another.

\section{Internal Pressure Signal:}

Casing vibration in a gas turbine engine, under test conditions, can be driven by two groups of forces [9]: 
a) forces from the rotational structural elements in the engine, such as the running gear, being transmitted through the casing/bearing attachments, and;

b) the aero/structure interaction of the internal pressure and the turbine casing

This second group of forces (b) is believed to be dominant in a real engine setup, and is indeed dominant in the experimental measurements shown in later sections of this paper. This second group of forces can then be broken down into a further subset of constituents being:

(i) interaction of the rotating pressure profile around each rotor blade (noting that this is only the case for unshrouded rotor blade setups)

(ii) propagation of acoustic waves inside the engine casing

(iii) pressure fluctuations on the casing wall due to turbulent and impulsive flows

It has been shown that the rotating pressure fluctuations, group (b)(i), are the most dominant excitation forces acting on the casing surface and thus drive its motion. Within this section of the paper the pressure force excitation which is developed from this group of forces will be derived. As stated, it is this group of forces which will be most dominant in the casing vibration measurements along with also being most prevalent in the measurements of the internal casing pressure signal.

\subsection{Internal pressure signal without blade vibration}

As air passes over the rotor blades, a harmonic pressure distribution forms around the blades; this first harmonic of the pressure profile which would develop around the rotor blades is shown in Figure 1, a schematic of a rotating bladed disk. Without any blade motion this harmonic pressure would rotate around at the engine input shaft speed, causing a fluctuating pressure force on the casing surface. This rotating pressure force will generally be a more complex shape than is shown in Figure 1, however without any loss of generality the pressure around the casing, without any blade motion, of any general pressure profile shape, for the $r^{\text {th }}$ blade, can be shown mathematically to be:

$$
P_{r}=\operatorname{Re}\left\{\sum_{i=0}^{\infty} A_{i} P e^{j i\left[\theta+\Omega(t)+\alpha_{r}+\gamma_{i}\right]}\right\}
$$

where $\mathrm{P}$ is the magnitude of the pressure, where $j=\sqrt{-1}$ and $A_{i}$ and $\gamma_{i}$ are the amplitude and phase of the corresponding Fourier series, $\alpha_{r}$ is shown in Figure 1 and $\theta$ is the angular position around the casing for any given datum. 


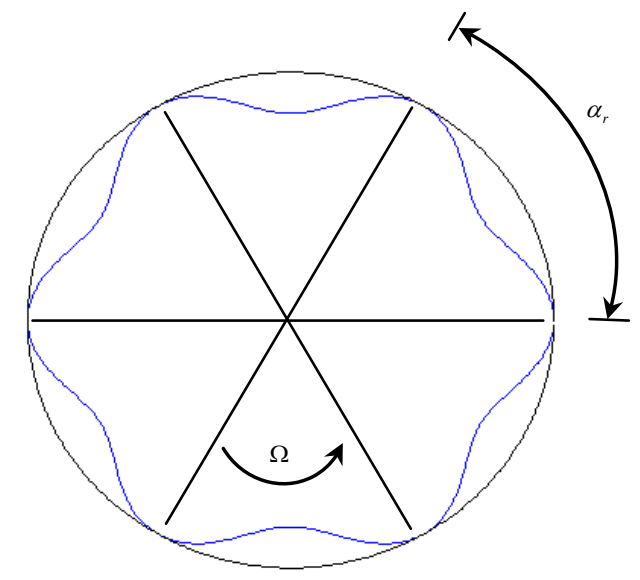

Figure 1 Schematic of simple first harmonic pressure distribution for a 6 bladed arrangement without blade vibration

\subsection{Rotor blade forces and vibration}

The above derivation in Equation (1), does not take into account any blade vibration; however, as blade vibration is inherent in the operation of a gas turbine engine, the forces which drive this blade vibration and the corresponding effect it has on the internal pressure signal derived in Equation (1) will now be shown.

Rotor blade vibration in a gas turbine develops from the rotor blades passing through the unsteady pressure profile trailing the upstream stator blade rows. This effect can be visualised with the aid of Figure 2, where a 1.5 stage turbine is shown schematically. It can be noted that as the rotor blades pass through the trailing wakes (and pressure fields) from the upstream stator blades a fluctuating pressure will be felt by the rotor blades, and this in turn provides an oscillating force on the blade. It should be noted that this oscillating pressure force is also applied to the stator blades; however, these forces on the stator blades have not been shown to be dominant (through moments at their fixations) in generating significant casing vibration [10]. Generally rotor blades are excited by the dominant discrete frequencies at stator passing frequency (SPF).

The excitation on the $r^{\text {th }}$ blade can now be mathematically described as:

$$
f(t)_{r}=F_{0}\left\{\sum_{q=0}^{\infty} C_{q} \cos \left[q\left(\Omega t+\gamma_{q}+\gamma_{r}\right)\right]\right\}
$$

The above force can be of any shape depending on the selected Fourier series co-efficients of $C_{q}, \gamma_{q}$ and where the phase offset $\gamma_{r}$ for the force on each rotor blade is given as:

$$
\gamma_{r}=\frac{2 \pi s(r-1)}{b}-\text { round }\left(\frac{s(r-1)}{b}\right) 2 \pi
$$


where the turbine stage contains ' $b$ ' number of rotor blades and 's' number of upstream stator blades.

(a)

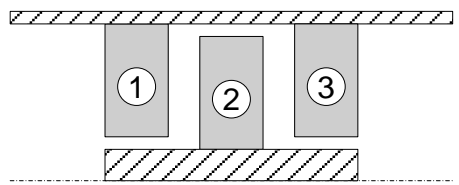

(1) IGV

(2) Rotor Blade

(3) Stator Blades (b)

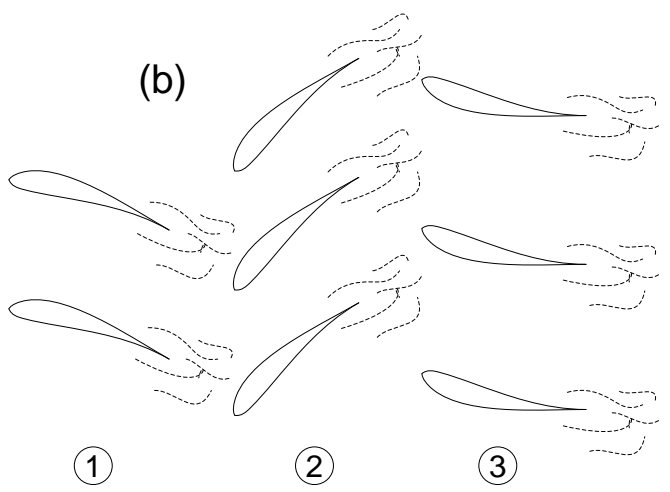

(1)

the Fourier coefficients can again be given values to describe any desired impulse shape. $g(t)$ is modelled as a white random variable with zero mean within this work, however in practice the turbulence will be somewhat band-limited and coloured. It should also be noted that this driving force should have a DC offset, which would create both a deterministic and stochastic portion to this signal, this DC offset is omitted in this derivation as only the stochastic portion of the pressure and casing vibration signals will be used in the later results for estimation of the turbine blade natural frequencies. For further information on derivation of the discrete portion of the signal see Ref. [7].

If the rotor blades are modelled as a single degree-of-freedom system, the spectrum of the blade motion will result from the multiplication of the force spectrum and the blade transfer function. The blade response will display the same second order statistics as that of the random variable $g(t)$. The spectrum of the blade motion will thus be stochastic in nature and in the power spectrum average the blade motion spectrum will be equal, in shape, to the blade transfer function. This process is shown schematically in Figure 3 with the instantaneous amplitude spectrum of the blade force, Equation (3), multiplied by the blade transfer function resulting in the instantaneous amplitude spectrum of the blade response. 

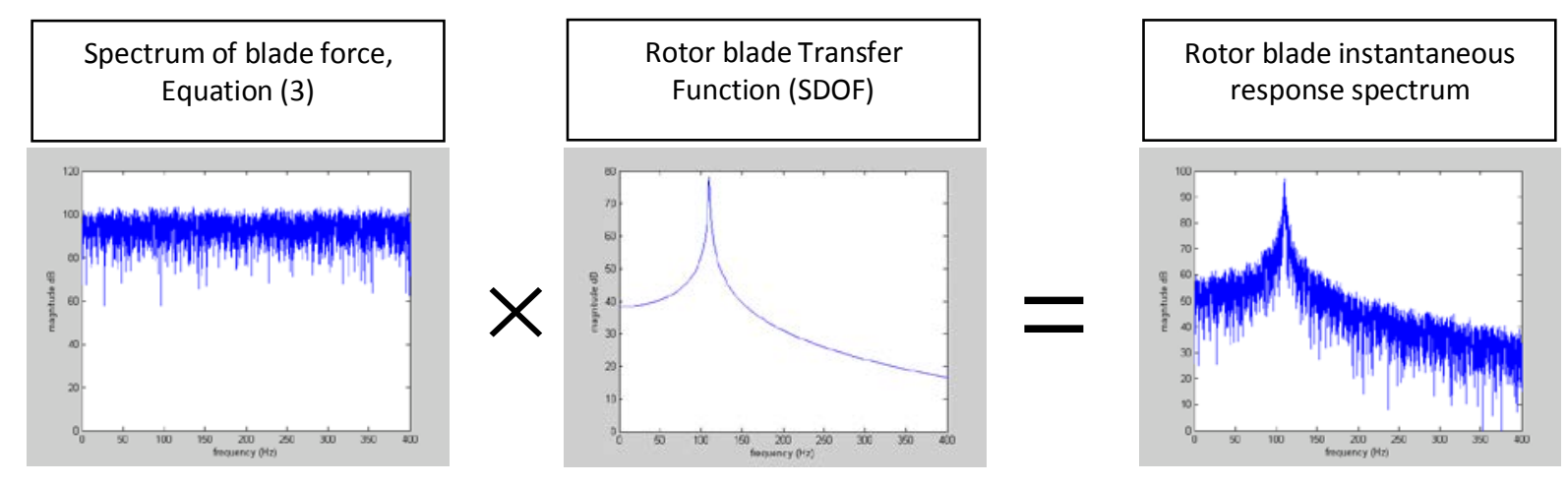

Figure 3 Schematic of the derivation the stochastic blade forced motion

\subsection{Internal pressure signal including blade motion}

Now that the blade response is known, the interaction the blade response has with the internal pressure can be developed. With the aid of Figure 4, it can be seen that the blade motion will change the rotating pressure profile which rotates around with the input shaft rotation speed, as the rotor blades oscillate around their equilibrium position. This will cause the internal pressure profile to be phase modulated by the blade vibration.

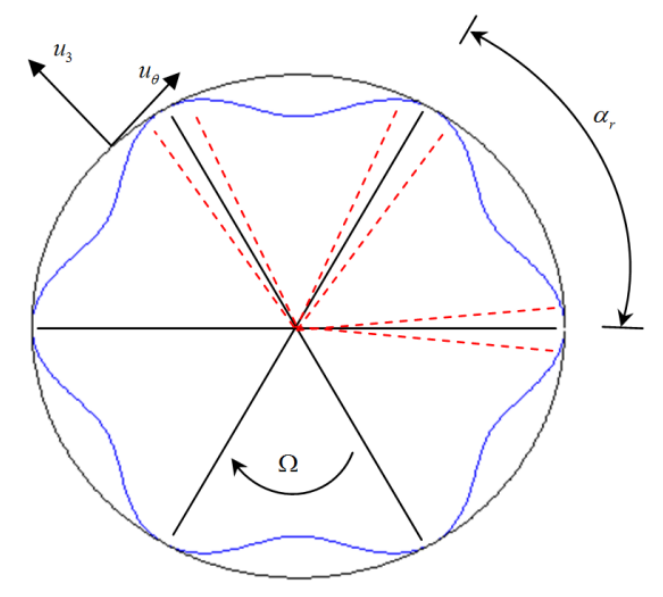

Figure 4 Schematic of simple first harmonic pressure distribution for a 6 bladed arrangement including blade motion

This phase modulation of the rotating pressure profile by the blade motion is simply added as a time dependent phase offset, due to blade motion, incorporated in the original pressure distribution derived in Equation (1). This yields the internal pressure signal with the inclusion of blade motion to be:

$$
P_{r}=\operatorname{Re}\left\{\sum_{i=0}^{\infty} A_{i} P e^{j i\left[\theta+\Omega t+\alpha_{r}+\gamma_{i}\right]} e^{j i[x(t) r]}\right\}
$$

The first exponential function in Equation (4) is deterministic and its spectrum will be a set of discrete harmonics at shaft speed $\Omega$. The spectrum of the second exponential function in 
Equation (4) will have the same form as the spectrum of the blade motion $x(t)_{r}$, shown schematically in Figure 3. The spectrum of this internal pressure signal will be created from the convolution of the spectrum of the deterministic rotating pressure signal and the stochastic blade motion. This process is shown schematically in Figure 5. It can be seen that the spectrum of the internal pressure signal is now made up of a series of narrow-band peaks located at shaft speed, $\Omega$, plus and minus the blade natural frequency. This is a significant result, and is the basis of the later experimental estimation of the blade natural frequencies, as it demonstrates that the stochastic portion of the internal pressure signal within the turbine engine will contain directly measurable information about blade natural frequencies. This pattern of narrow band peaks is shown in the instantaneous internal pressure spectrum from the later experimental measurements in Figure 6 (a).

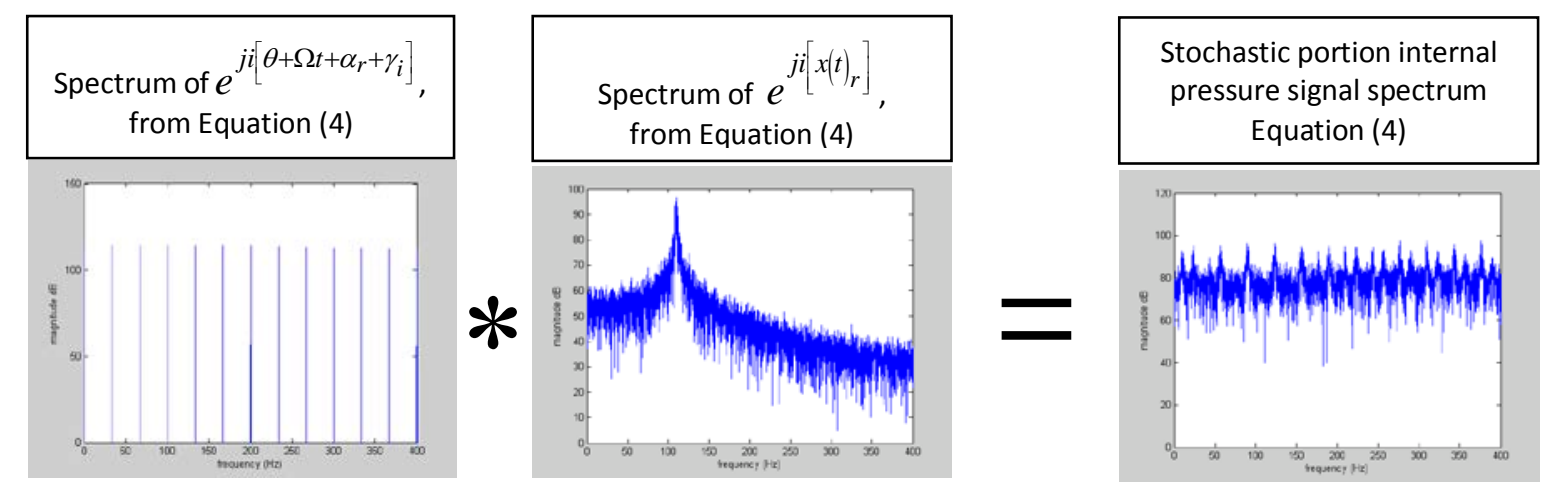

Figure 5 Schematic of the derivation of internal pressure spectrum
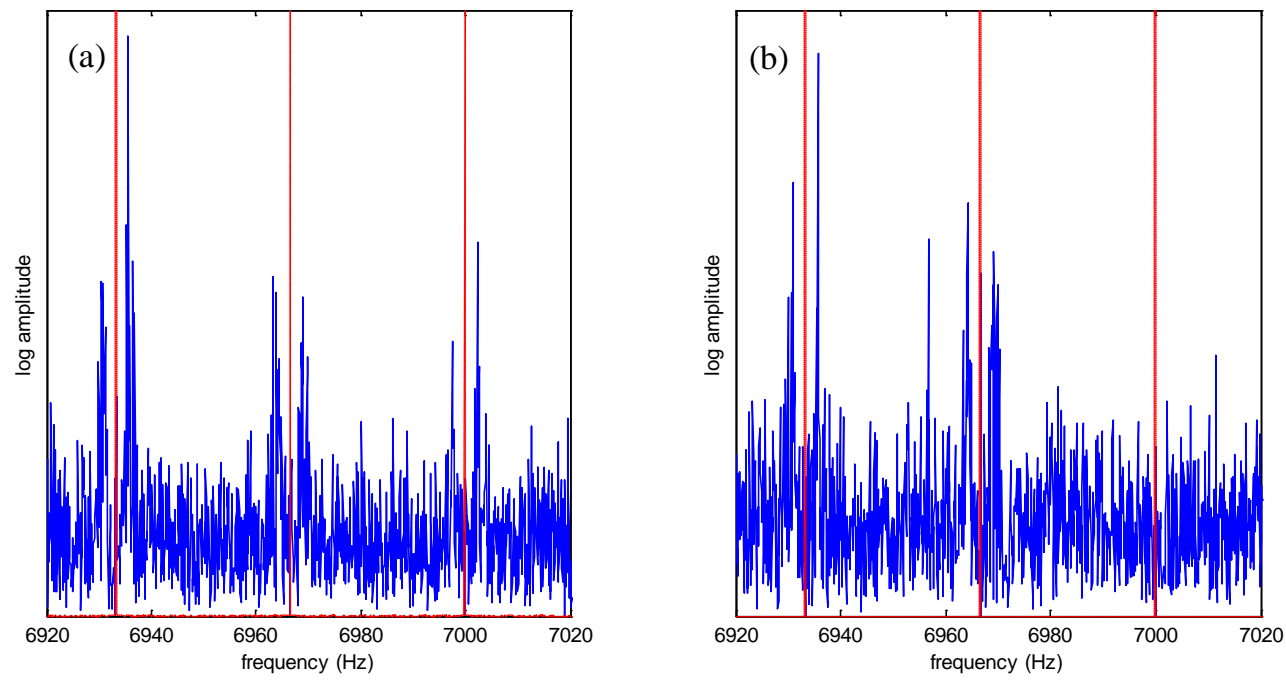

Figure 6 Stochastic pressure signal from the test rig at 2000rpm, notice the three sets of narrow band peaks centred around harmonics of the shaft speed (the dotted vertical lines) spaced at $33.3 \mathrm{~Hz}$.

(a) internal pressure (b) casing response

The casing vibration signal will also display all the same properties as those of the internal pressure signal. The casing will act as a linear time invariant (LTIV) filter, and will alter the 
magnitude of the signal and add some underlying structural resonances, but the sets of narrow band peaks will still be present and can be seen in the casing vibration response in Figure 6 (b). Derivation of the response of a circular ring under the influence of a deterministic rotating pressure force can be found in Ref. [11] and under stochastic forces in Ref. [7], but will not be entered into within this paper as it has been shown the transmission simply acts as a LTIV filter.

\section{Experimental Setup:}

In order to experimentally estimate the natural frequency of turbine blades from casing pressure and vibration measurements, a simplified test rig was employed, which introduced blade vibration excitation with a single row of rotor blades. This test rig consisted of a nineteen (19) flat bladed disk arrangement, driven by an electric motor run at speeds up to $2400 \mathrm{rpm}$. A toroidal ring in front of the bladed disk, supplied with high pressure air, had six (6) air jets which emitted high velocity air to act like the upstream trailing edge wakes through which turbine blades have to pass during their rotation, and which drives blade vibration. These six (6) air jets therefore excite the blades at multiples of shaft speed, and contain a significant turbulent component. The test rig can be seen, without the external protective housing, in Figure 7. The placement of a microphone, for high speed pressure measurement, and an accelerometer on the rotor casing are also highlighted in Figure 7. The microphone was flush mounted with the inner casing surface and the accelerometer measured casing vibration in the vertical plane. It can also be noted that the casing is of a nonsymmetric semi-circular shape. It is not believed that this would make any fundamental difference to the measurements obtained from those taken on a fully circular casing of a commercial turbine, in consideration of the fact that the absolute pressure is very low.

To simulate a potential degradation in one of the turbine blades, resulting in a reduced blade stiffness and thus lower natural frequency, one of the blades was replaced by a thinner blade. Measurements were then recorded again with this arrangement of one simulated faulty blade. The original 19 blades had the nominal blade dimensions of 100(L)x50(W)x1.2(T)mm with the simulated faulty blade having a reduced thickness of $0.9 \mathrm{~mm}$. Table 1 contains the first and second bending mode natural frequencies measured during a stationary bench test, with the mean value of the natural frequencies shown for all 19 blades of $1.2 \mathrm{~mm}$ thickness. 


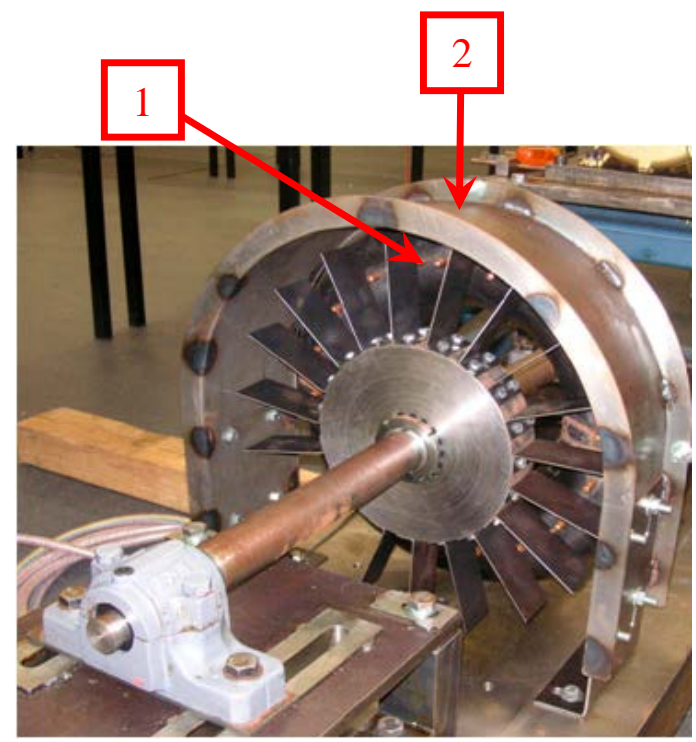

Figure 7 Experimental test rig with 19 blades. (1) 6 air jets located on a toroidal ring which is supplied with high pressure air. (2) location of microphone and accelerometer mounting

Table 1 Measured second bending mode natural frequencies from a stationary bench test

\begin{tabular}{lll}
\hline Nominal blade thickness & $1.2 \mathrm{~mm}$ & $0.9 \mathrm{~mm}$ \\
\hline $1^{\text {st }}$ Bending natural frequency & $117.4 \mathrm{~Hz}^{*}$ & $69 \mathrm{~Hz}$ \\
$2^{\text {nd }}$ Bending natural & $726 \mathrm{~Hz}^{*}$ & $432 \mathrm{~Hz}$ \\
frequency & &
\end{tabular}

*Mean natural frequency of all 19 blades measured

After capture, all vibration and pressure measurements were post-processed in the same fashion. This process is outlined in Figure 8, with a 10 second record being captured whilst the test rig was run at a constant speed. The measurements were then order tracked (phase resampled) with the use of a once per-rev tachometer pulse signal which was also recorded during measurement. The phase resampled signal was then synchronously averaged to separate the discrete and random portions of the pressure and vibration signals. Finally, in order to give a robust estimate of the blade natural frequency an ensemble average of the vibration and pressure spectrum was undertaken with the bandwidth across one shaft rotation, spanning from 0.5 to 1.5 multiples of shaft speed. This process is illustrated in Figure 9 for the rotational speed of $1200 \mathrm{rpm}(20 \mathrm{~Hz})$, where the spectrum is split into sections, $20 \mathrm{~Hz}$ in width, which span from 10-30, 30-50, 50-70 Hz etc, and then ensemble averaged. This gives a robust estimate of the spacing between the narrow band peaks in the residual vibration and pressure signals to then use as an estimate of the turbine blade natural frequency. 


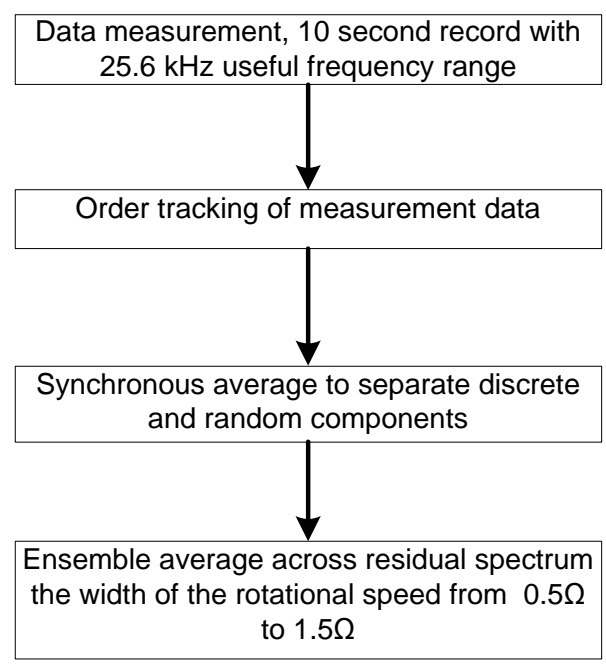

Figure 8 Flow chart of measurement Post-processing

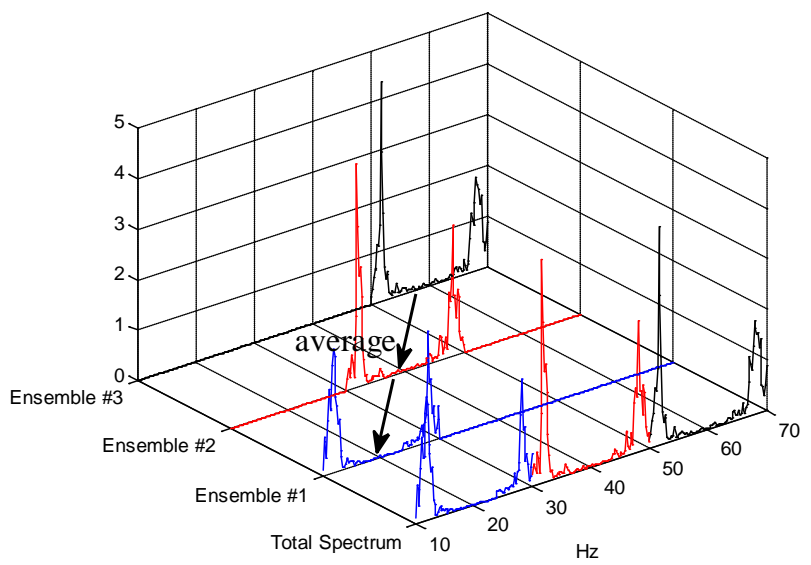

Figure 9 Ensemble averaging schematic. Process shown for the 1200rpm shaft speed case.

\section{Analytical estimation of blade natural frequencies}

Firstly the estimation of turbine blade natural frequencies is conducted on the analytically generated internal pressure signal, given in Equation (4), before verifying with the results obtained from experimental measurements taken on the test rig, as outlined above.

As the set of narrow band peaks lie at multiples of shaft speed plus and minus the blade natural frequency, $q \Omega \pm$ Blade nat.f where $q$ is any positive integer number, then as long as the blade natural frequency is known to within \pm half of the shaft speed, the distance between these narrow band peaks can be used to estimate the blade natural frequency which is being excited. Figure 10 shows schematically how the narrow band peaks are located around the harmonics of shaft speed. 


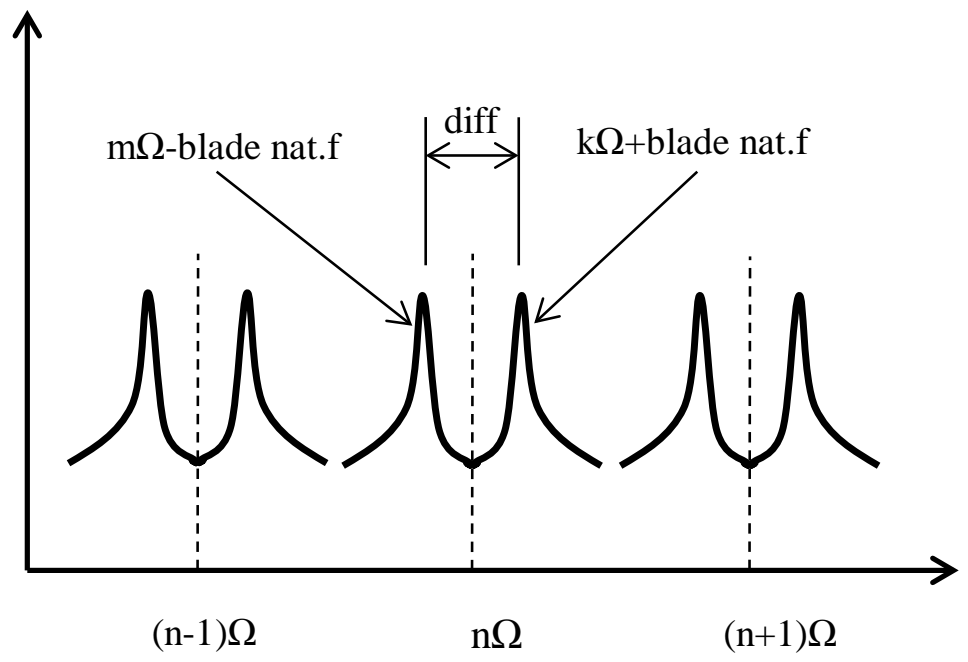

Figure 10 Schematic of the relationship of the narrow band peak harmonics and blade natural frequency With the aid of Figure 10 the spacing between the narrow band peaks (diff) and the blade natural frequency can be derived:

$$
\begin{aligned}
& \text { diff }=(k \Omega+\text { blade nat. } f)-(m \Omega-\text { blade nat. } f) \\
& \text { Blade nat. } f=\frac{\text { diff }+(m-k) \Omega}{2}
\end{aligned}
$$

where $k m$ and $n$ are positive integers and $k<m$

which results in the following relationship,

$$
\text { Blade nat. } f=\frac{\text { diff }+q \Omega}{2}
$$

With the use of Figure 6, which contains narrow band peaks separated approximately by 16.5 $\mathrm{Hz}$ and the shaft rotational speed of 1200rpm (20 Hz), an example of how to use Equation (5) to estimate the blade natural frequency will now be given.

According to Equation (5) a number of natural frequency values would satisfy the difference (diff) value of $16.5 \mathrm{~Hz}$ and the rotational speed of $20 \mathrm{~Hz}$ as $q$ can be any integer value. With the knowledge of an approximate value of blade natural frequency, say $726 \mathrm{~Hz}$ for the second bending mode measured in a stationary bench test as given in Table 1, then Equation (5) will yield the values of:

$$
\text { Blade nat. } f=\frac{16.5+q \times 20}{2}=718,728 \mathrm{~Hz} \text { for } q=71,72
$$


As the blade natural frequency should increase with rotational speed then the estimate of the blade natural frequency would be given as $728 \mathrm{~Hz}$. Thus from the knowledge of shaft rational speed, measurement of the distance between the sets of narrow band peaks and knowledge of the blade natural frequency to within \pm half the rotational shaft speed, the blade natural frequency can be estimated from internal pressure or casing vibration measurements. Note that this uncertainty of \pm half the rotational shaft speed applies equally to the BTT method, since arrival times are measured only once per shaft revolution.

Now setting all the 19 blade natural frequencies to the values as given in Table 2, the analytically simulated internal pressure signal, given by Equation (4), is developed for rotational speeds of 1200 and 2000rpm. The results of the ensemble averaged pressure spectrum can be seen in Figure $11(\mathrm{a}-\mathrm{b})$ respectively. It is shown that the spacing between the narrow band peaks is at 16.5 and $4.9 \mathrm{~Hz}$ respectively. (Note in the ensemble averaged spectrum the centre frequency is always a multiple of the shaft speed. However for consistency the lowest frequency is set to zero in Figures 10, 12 and 13. See Figure 9 for clarification of the ensemble averaging process).

Additionally the simulated faulty blade was also modelled analytically, with the reduced natural frequency of one blade with a thickness $0.9 \mathrm{~mm}$. The ensemble averaged internal pressure spectrum can be seen in Figure $11(\mathrm{c}-\mathrm{d})$. The spacing between the narrow band peaks is given in Table 2. It can be seen that the response of the single blade with reduced thickness dominates the response with almost no visible response from the other 18 thicker blades being present in the ensemble averaged spectrum. Although this seems somewhat nonintuitive, as the 18 blades should add together to dominant the pressure spectrum over the single faulty blade, the reduced stiffness of the single thinner blade has significantly increased its compliance and thus vibration response. This reduced stiffness due to the decrease in blade thickness can be calculated, as below, to be approximately 3.8 times less stiff than the original blades, and thus dominates the response spectrum.

$$
\begin{gathered}
\omega_{n 1}=\sqrt{\frac{k_{e q 1}}{m_{e q 1}}}=728 \mathrm{~Hz} \\
\omega_{n 2}=\sqrt{\frac{k_{e q 2}}{m_{e q 2}}}=432 \mathrm{~Hz} \\
0.75 m_{e q 1}=m_{e q 2} \\
\therefore \frac{k_{e q 1}}{k_{e q 2}}=\frac{\omega_{n 1}^{2}}{0.75 \omega_{n 2}^{2}} \approx 3.8
\end{gathered}
$$


Table 2 Blade natural frequencies used in analytically derived simulated pressure signal

\begin{tabular}{lcc|cc}
\hline $\begin{array}{l}\text { Rotational } \\
\text { speed }\end{array}$ & \multicolumn{2}{c|}{ 19 identical blades } & \multicolumn{2}{c}{ Simulated single faulty blade } \\
\hline & $\begin{array}{c}\text { Natural } \\
\text { frequency }\end{array}$ & $\begin{array}{c}\text { Narrow band } \\
\text { peak spacing }\end{array}$ & $\begin{array}{c}\text { Natural } \\
\text { frequency }\end{array}$ & $\begin{array}{c}\text { Narrow band } \\
\text { peak spacing }\end{array}$ \\
$\mathbf{1 2 0 0} \mathbf{~ r p m}$ & $728 \mathrm{~Hz}$ & $16.5 \mathrm{~Hz}$ & $432 \mathrm{~Hz}$ & $16.5 \mathrm{~Hz}$ \\
$\mathbf{2 0 0 0} \mathbf{~ r p m}$ & $733 \mathrm{~Hz}$ & $4.85 \mathrm{~Hz}$ & $441.5 \mathrm{~Hz}$ & $15.8 \mathrm{~Hz}$ \\
\hline
\end{tabular}
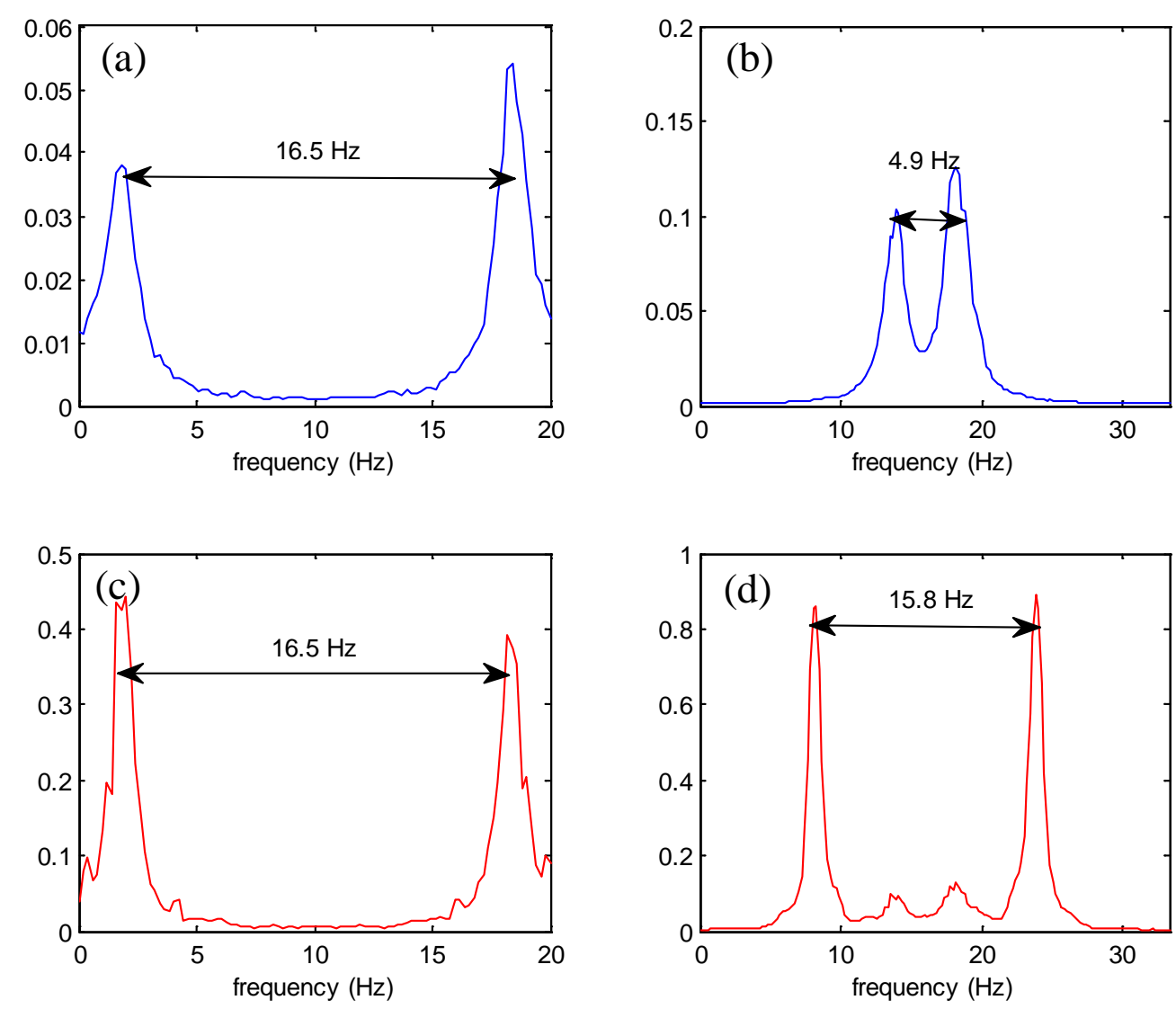

Figure 11 Ensemble spectral average of analytically derived casing pressure residual power spectrum. (a) \& (c) 1200rpm no-fault and with faulty blade respectively. (b) \& (d) 2000rpm with no-fault and with faulty blade respectively.

\section{Experimental estimation of turbine blade natural frequencies}

To validate the above analytical simulations and to estimate the turbine blade natural frequencies experimentally, the test rig was run at 3 different speeds, being 1200, 2000, 2400rpm. Pressure and casing vibration measurements were recorded. A typical Power Spectral Density plot for the separated discrete and random portions of the casing and vibration signals can be seen in Figure 12 for the 2400rpm measurement. The pressure and vibration signals display the same signal properties with some differences in the magnitude of the spectral values due to the structural transfer function of the casing for the vibration 
measurements. A set of discrete harmonics are present at multiples of shaft speed, and it can be seen that there is a set of underlying broadband peaks which modulate these discrete harmonics, particularly noticeable around the $5320 \mathrm{~Hz}$ harmonic of shaft speed. These underlying narrow band peaks were discussed above in the generation of the internal pressure signal and come from the broadband excitation of the rotor blade natural frequencies. Estimation of the blade natural frequency can be made from the observation of the distance between these narrow band peaks.
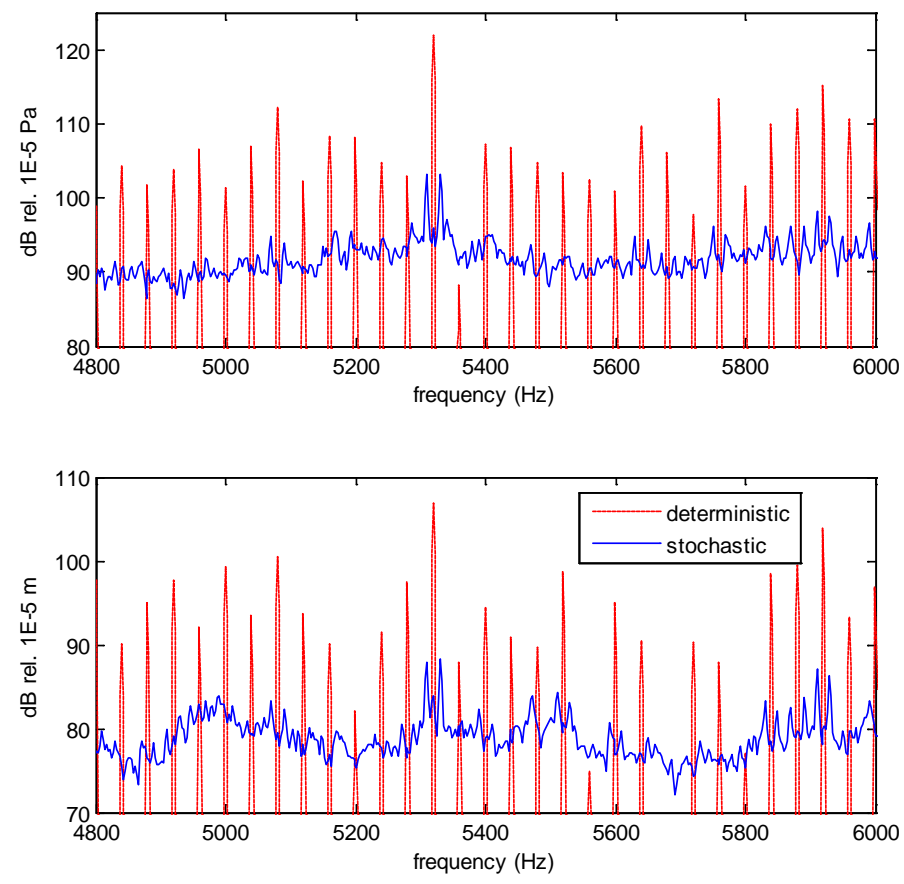

Figure 12 PSD of pressure (top) and vibration (bottom) measurements on the test rig.

It can be seen in Figure 12 that not all harmonics of shaft speed have noticeable narrow band peaks around them, so in order to better estimate the narrow band peak spacing an ensemble average of the raw residual pressure and vibration spectrum was undertaken. This also allows an average of the entire spectrum to be analysed without loss of spectral resolution. The results of the ensemble averaged pressure and casing spectra are shown in Figure 13. Figure 13 (a) and (e) show the pressure and casing vibration when the air jets are off, such that very little blade vibration would be occurring. As would be expected, no sets of narrow band peaks are seen to exist. All other plots in Figure 13 show a set of narrow band peaks with the exception of Figure 13 (d) where it is believed that due to the low rotational speed not enough pressure existed to significantly excite the casing response. The narrow band peak spacing and estimated natural frequencies from the experimental measurements are given in Table 3, and correspond well with the analytically derived results given in Table 2.

Unlike the analytical derivation of the pressure spectrum in the previous section where a single degree of freedom system was used in the blade response model, the turbine blades on the test rig exhibit many modes of vibration. The ensemble averaged spectrum only seems to 
contain a single set of narrow band peaks with a spacing which indicates that only the second bending mode of vibration is being excited. It was thought that the first two vibration modes should dominate the response spectrum due to their higher modal participation factors, and as was shown in the previous section, the blades and modes which have the highest vibration response will greatly dominate the response spectrum. The modal participation factor of the first and second mode are relatively similar, and the lack of response of the first mode is not yet fully understood, but is almost certainly due to the fact that the pressure fluctuations are related to the acceleration of the blade rather than its displacement, and this gives a much higher weighting to the second bending mode in the response spectrum. Further work will be needed to investigate fully the reason for the low response of the first bending mode, but it is possibly related to the frequency content of the exciting turbulence.
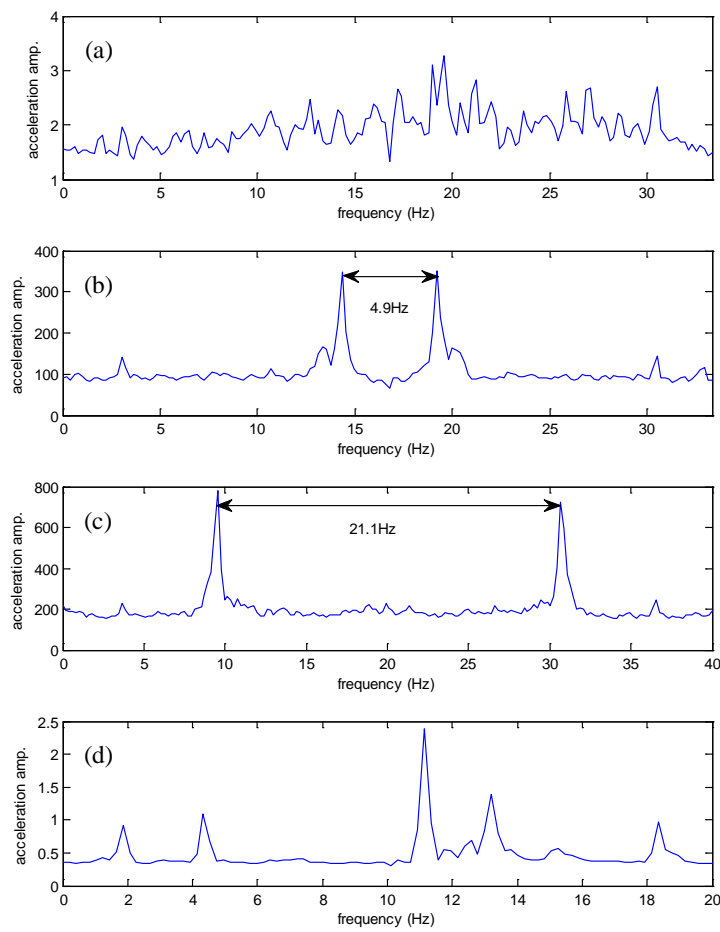
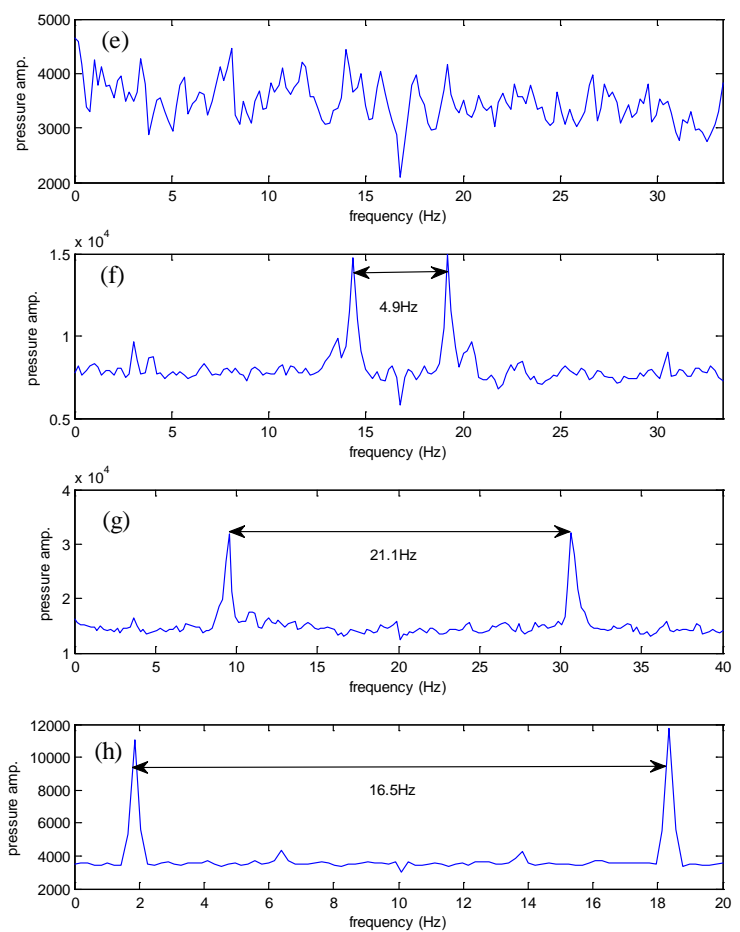

Figure 13 Ensemble spectral average of residual power spectrum. (a) \& (e) 2000rpm air jets off. (b) \& (f) 2000 rpm with air jets on. (c) \& (g) 2400rpm with air jets on. (d) \& (h) 1200 rpm with air jets on. All measurements taken on experimental test rig.

Table 3 Estimated natural frequencies for the test rig at different running speeds

\begin{tabular}{lll}
\hline Rotational speed & Narrow band peak spacing & $\begin{array}{l}\text { Estimated natural } \\
\text { frequency }\end{array}$ \\
\hline 1200rpm & $16.5 \mathrm{~Hz}$ & $728.25 \mathrm{~Hz}$ \\
$\mathbf{2 0 0 0 r p m}$ & $4.9 \mathrm{~Hz}$ & $733 \mathrm{~Hz}$ \\
$\mathbf{2 4 0 0 r p m}$ & $21.1 \mathrm{~Hz}$ & $749 \mathrm{~Hz}$ \\
\hline
\end{tabular}




\section{Experimental simulation of faulty turbine blade}

To simulate a faulty turbine blade, one of the 19 blades in the test rig was replaced by a blade of a reduced thickness, of $0.9 \mathrm{~mm}$ compared to the $1.2 \mathrm{~mm}$ thickness for the other 18 blades.

The test rig was now run at 1200 and 2000rpm and the pressure and casing vibration measurements recorded. The ensemble averaged pressure spectrum for the two speeds can be seen in Figure 14. The sets of narrow band peaks can be seen to have shifted for the ensemble spectrum when the faulty blade is present, this results in the estimated blade natural frequencies which are found in Table 4. These natural frequency estimates correspond well with the increase in natural frequency which would be expected due to the rotational speed from the measured stationary natural frequency for the thinner blade of $432 \mathrm{~Hz}$.
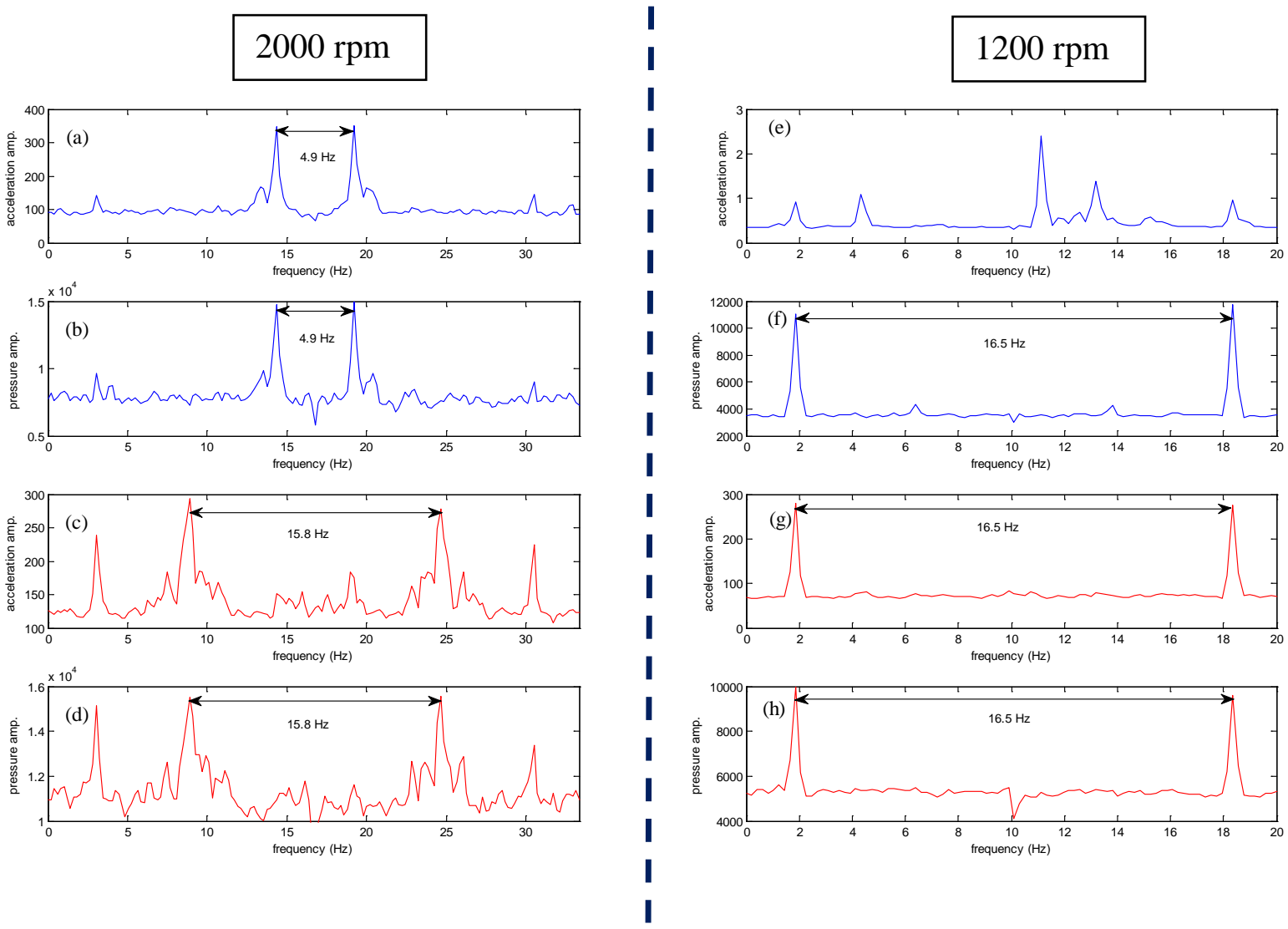

Figure 14 Ensemble spectral average of residual power spectrum. (a) \& (b) 2000rpm no faulty blade. (c) \& (d) 2000rpm with faulty blade. (e) \& (f) 1200rpm no faulty blade. (g) \& (h) 1200rpm with faulty blade

Table 4 Estimated natural frequencies for the test rig at different running speeds with a simulated faulty blade

\begin{tabular}{lcc}
\hline Rotational speed & Narrow band peak spacing & $\begin{array}{c}\text { Estimated faulty blade } \\
\text { natural frequency }\end{array}$ \\
\hline 1200rpm & $16.5 \mathrm{~Hz}$ & $438 \mathrm{~Hz}$ \\
$\mathbf{2 0 0 0 r p m}$ & $15.8 \mathrm{~Hz}$ & $441.5 \mathrm{~Hz}$ \\
\hline
\end{tabular}




\section{Conclusions:}

Traditionally, turbine blade natural frequencies cannot be measured in practice whilst the engine is running, and no form of non-contact measurement is available. Within this paper, the simulated internal pressure signal within a turbine engine has been derived with the inclusion of the effects of blade vibration on this internal pressure signal. It was shown that this internal pressure signal can contain sets of narrow band peaks which are spaced at multiples of shaft speed \pm blade natural frequency, therefore giving a measureable parameter to estimate the blade natural frequency of the internal rotating blades whilst the engine is in operation and running at a constant speed. The casing vibration response was also shown to contain the same signal properties as the internal pressure signal after it had passed through a LTIV filter, thus allowing an externally mounted accelerometer to measure the rotor blade natural frequencies. Results were shown for analytically derived internal pressure signals and the estimation of blade natural frequencies from a SDOF model of the blade response from the ensemble averaged pressure spectrum. The natural frequency of a single simulated faulty blade (reduced natural frequency) was then also estimated. The estimation of these blade natural frequencies relies on knowledge of the natural frequency to within \pm half of the shaft speed, which is also a limitation on the current BTT method. The analytical derivation of the internal pressure signal and estimation of the blade natural frequencies was then verified from measurements taken on a simplified test rig. The estimation of the blade natural frequencies was also achieved with the use of casing vibration measurements including successful estimation with a single simulated faulty blade (with reduced thickness). The work and results within this paper present the first non-contact method for determining turbine blade natural frequencies from measurements taken whilst the engine is running at a single operating speed.

\section{Acknowledgment:}

Grateful acknowledgment is made for the partial financial assistance given by the Australian Defence Science and Technology Organisation, through the Centre of Expertise in Helicopter Structures and Diagnostics at UNSW, during the collection of data for this work.

\section{References:}

1. Meher-Homji, C.B. Blading vibration and failures in gas turbines part A: blading dynamics \& the operating environment. 1995. Houston, TX, USA: ASME, New York, NY, USA.

2. Knappett, D. and J. Garcia, Blade tip timing and strain gauge correlation on compressor blades. Proceedings of the Institution of Mechanical Engineers, Part G (Journal of Aerospace Engineering), 2008. 222(G4): p. 497-506.

3. Zablotskiy, I. and Y. Korostelev, Measurement of Resonance Vibrations of Turbine Blades with the Elura Device Energomashinostroneniye, Nr. 2, 1970: p. 36-39.

4. Heath, S. and M. Imregun, Improved single-parameter tip-timing method for turbomachinery blade vibration measurements using optical laser probes. International Journal of Mechanical Sciences, 1996. 38(10): p. 1047-1058. 
5. Forbes, G.L. and R.B. Randall. Simulated Gas Turbine Casing Response to Rotor Blade Pressure Excitation. in 5th Australasian Congress on Applied Mechanics. 2007. Brisbane, Australia.

6. Forbes, G.L. and R.B. Randall, Non-contact gas turbine blade vibration measurement from casing pressure and vibration signals, in IFToMM-Rotordynamics 20102010: Seoul, Korea.

7. Forbes, G.L., Non-contact gas turbine blade vibration monitoring using internal pressure and casing response measurements, 2010, The University of New South Wales: PhD Dissertation. p. 211.

8. Rao, A.R. and B.K. Dutta, Non Intrusive Method of Detecting Turbine Blade Vibration in an Operating Power Plant, in Proceedings of ISMA 2010 including USD 20102010: Leuven, Belgium.

9. Mathioudakis, K., E. Loukis, and K.D. Papailiou. Casing vibration and gas turbine operating conditions. 1989. Toronto, Ont, Can: Publ by American Soc of Mechanical Engineers (ASME), New York, NY, USA.

10. Cumpsty, N.A., Compressor Aerodynamics2004: Krieger Publishing Company.

11. Forbes, G.L. and R.B. Randall, Resonance phenomena of an elastic ring under a moving load. Journal of Sound and Vibration, 2008. 318(4-5): p. 991-1004. 